\title{
B019
}

\section{Laterally Constrained Inversion of Fixed-Wing Frequency-Domain AEM Data}

\author{
E. Tartaras* (British Geological Survey) \& D. Beamish (British Geological \\ Survey)
}

\section{SUMMARY}

New high-resolution airborne geophysical surveys of the UK, undertaken with the system developed under the Joint Airborne-geoscience Capability programme, established between the Geological Survey of Finland and the British Geological Survey, will provide large 4-frequency airborne electromagnetic data sets. These data sets will be used to characterise the conductivity distribution of the subsurface for environmental and exploration purposes. To invert these large data sets in a fast and robust manner we have developed "LC1DINV", a laterally constrained one-dimensional inversion algorithm. This algorithm inverts simultaneously for all observation points along a profile and regularises the inverse problem by requiring that differences between model parameters at adjacent points be small. We use the conjugate gradient method for minimising the data misfit subject to the lateral constraints and a priori model terms. We have inverted 4-frequency data obtained over Suurpelto, a test area in southern Finland, characterised by conductive clays overlying a highly resistive granitic shield. The results show that LC1DINV can successfully locate the depth extent and variations of the clays. Comparison of these results with those obtained with two other types of inversion shows that LC1DINV produces well-defined layer boundaries and laterally smooth cross-sections. 


\section{Near Surfocentul}

\section{Introduction}

New high-resolution airborne geophysical coverage of the UK is to be provided by a fixedwing surveying system developed under the Joint Airborne-geoscience Capability (JAC) programme, established between the Geological Survey of Finland (GTK) and the British Geological Survey (BGS). The new system is developed from the existing GTK facility described by Poikonen et al. (1998). These surveys will acquire large multi-frequency airborne electromagnetic (AEM) data sets in order to characterise the conductivity distribution of the subsurface for environmental and exploration purposes. This paper describes the laterally constrained one-dimensional (1-D) inversion algorithm "LC1DINV" that we have developed for inverting large AEM data sets in a fast and robust manner.

\section{Frequency-domain AEM inversion methods}

AEM surveys produce large data sets that require fast and stable inversion tools. Industry standard practice for interpretation of frequency-domain AEM data is to use half-space inversions, i.e. apparent resistivity transformations, that invert for the resistivity of a halfspace and its depth below the receiver (Huang and Fraser, 1996). These transformations are fast and stable and they can produce a good representation of horizontal resistivity variations. However, they don't allow detection of resistivity variations with depth and suffer in areas where the resistivity model departs significantly from that of a half-space. In order to distinguish vertical resistivity variations and obtain a formal measure of model validity one can use a multi-layer 1-D inversion (Beard, 2000; Constable et al., 1987; Fitterman and Deszcz-Pan, 1998; Paterson and Redford, 1986; Sengpiel and Siemon, 1998). These inversions are non-unique because they typically solve a heavily underdetermined problem. They therefore employ additional constraints to stabilise the problem, such as the MarquardtLevenberg regularisation or smoothness constraints. The former inversion type leads to laterally "noisy" looking cross-sections, whereas the latter type (called "Occam" inversion) manages to fit the data very well, but it produces very smooth sections where formation boundaries are difficult to distinguish.

\section{Laterally constrained conjugate gradient inversion algorithm}

To overcome the aforementioned problems we have developed a laterally constrained inversion (Auken et al., 2002; Smith et al., 1999). In this type of inversion we invert simultaneously for several observation points and we require that lateral changes in the model parameters from one observation point to the next be small. Thus, we retain the ability to distinguish different layers while obtaining laterally smooth cross-sections. Moreover, we may, if we have such information, require that our model be close to an a priori model, $m_{\text {apr }}$. Mathematically the above requirements mean that instead of just trying to minimise the data misfit, we try to minimise the parametric functional $P(m)=\phi(m)+\alpha \operatorname{Sm}(m)+\beta \operatorname{Sr}(m)$, where $\alpha$ and $\beta$ are regularisation parameters, $\phi$ is the misfit functional and $S m$ and $S r$ are stabilising functionals:

$$
\begin{gathered}
\phi(m)=W_{d}\left\|d_{p}-d\right\|^{2}, \\
S m(m)=W_{m}\left\|m-m_{a p r}\right\|, \\
\operatorname{Sr}(m)=W_{m}\|R m\| .
\end{gathered}
$$

$W_{d}$ and $W_{m}$ are data and model weighting matrices respectively, and $R$ is the roughening matrix that implements the lateral constraints. 
The roughening matrix is applied to the vector of model parameters, $m$. It contains 1 and -1 's for the constrained parameters and 0 at all other places. For example the first row of the roughening matrix has 1 in the first column that corresponds to the first parameter, i.e., the resistivity of the first layer, for observation point \#1 and -1 in the column that corresponds to the same parameter but for observation point \#2. The same is true for the columns that correspond to the other parameters (thickness of the first layer, resistivity of the second layer and so forth). The second row has 1 and -1 for the same parameters but for observation points $\# 2$ and \#3. Thus matrix $\mathrm{R}$ has a banded form:

$$
R=\left[\begin{array}{cccccccccc}
1 & 0 & \cdots & 0 & -1 & 0 & \cdots & 0 & 0 & 0 \\
0 & 1 & 0 & \cdots & 0 & -1 & 0 & \cdots & 0 & 0 \\
\vdots & & & & & \vdots & & & & \vdots \\
0 & 0 & 0 & \cdots & 0 & 1 & 0 & \cdots & 0 & -1
\end{array}\right]
$$

The effect of the roughening matrix is that it penalises large differences between parameters at adjacent points.

We use the conjugate gradient (CG) method (Hestenes and Stiefel, 1952) to find the minimum of the parametric functional. The CG method has the well-known advantage that it does not require computation of the inverse of the Hessian. Moreover, since the step size $k$ for the model update is calculated using the conjugate directions for all points, this method is inherently stable and does not require regularisation even when lateral constraints are not used; localised data anomalies caused by cultural noise that do not show lateral continuity along the profile are "ignored" during the model update process.

\section{Inversion of Suurpelto 4-frequency AEM data}

The current GTK fixed-wing AEM system is 2-frequency (Poikonen et al., 1998), but a new 4-frequency vertical coplanar system $(0.9,3,12$ and $24.5 \mathrm{kHz})$ has been developed under the JAC programme, established between GTK and BGS.

The new 4-frequency system was tested in November 2005 over the Suurpelto test area, a small area near Espoo in southern Finland. The Suurpelto area comprises mainly agricultural land and woodland and is now under development planning for new housing. The surficial geology is dominantly clays with some organic soils on the top. In bedrock troughs, the thickness of the overburden sediments can reach $25-30 \mathrm{~m}$. The test site is characterised by a zone of high electrical conductivity associated with saline and sulphide bearing clays. Laboratory electrical conductivity results of the clays show conductivities in excess of 200 $\mathrm{mS} / \mathrm{m}$ (Kurimo et al. 2006). The bedrock is highly resistive granitic shield.

Figure 1 shows a comparison of results from 3 different types of inversion for one flight line (Line 06). The 3 inversions are a) Marquardt, two layer inversion; b) Occam 61 layer inversion (Constable et al., 1987); c) LC1DINV two layer inversion. All the inversions extend to $60 \mathrm{~m}$ in depth. Figure 2 shows a series of successive conductivity cross-sections obtained with LC1DINV. The survey lines are parallel and spaced $200 \mathrm{~m}$ apart. It is clear that the laterally constrained inversion successfully recovers the depth extent and variations of the clays, while ignoring cultural noise and producing laterally smooth conductivity crosssections. 


\section{Near Surfece}

Marquardt: 2 layer

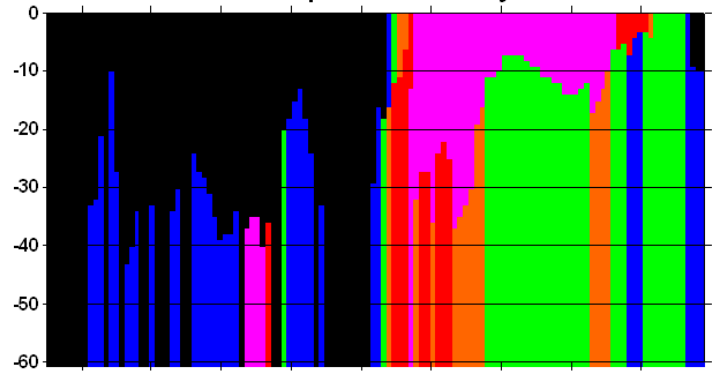

\begin{tabular}{|l|}
\hline Con $(\mathrm{mS} / \mathrm{m})$ \\
0.5 to 4 \\
4 to 8 \\
8 to 16 \\
16 to 32 \\
32 to 64 \\
64 to 500.1
\end{tabular}

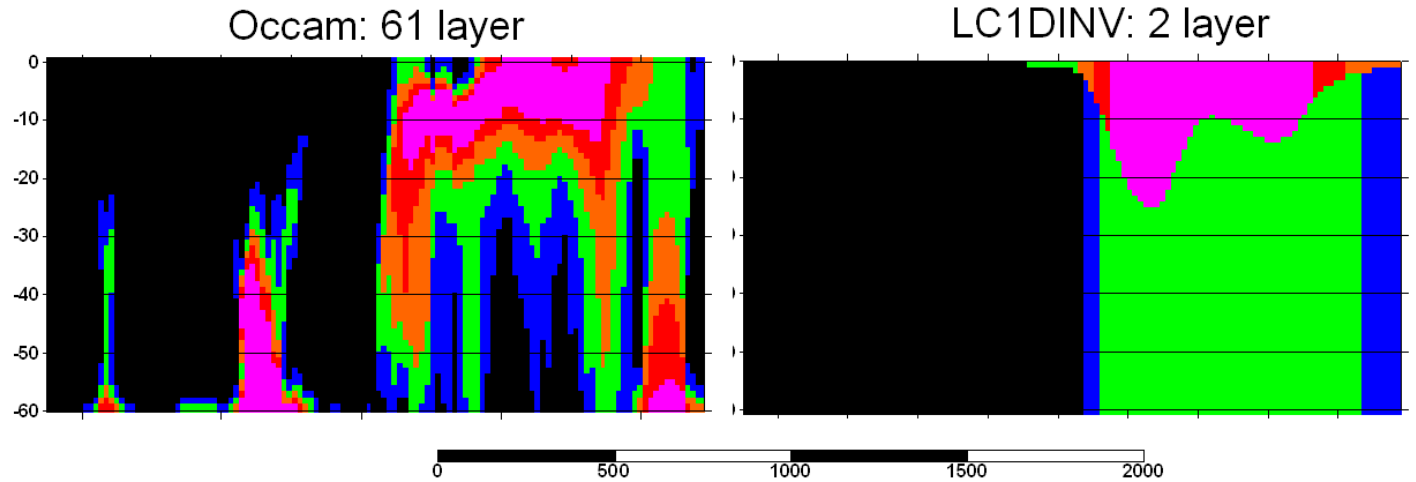

Figure 1. Comparison of results obtained with 3 different inversion methods for one Suurpelto line

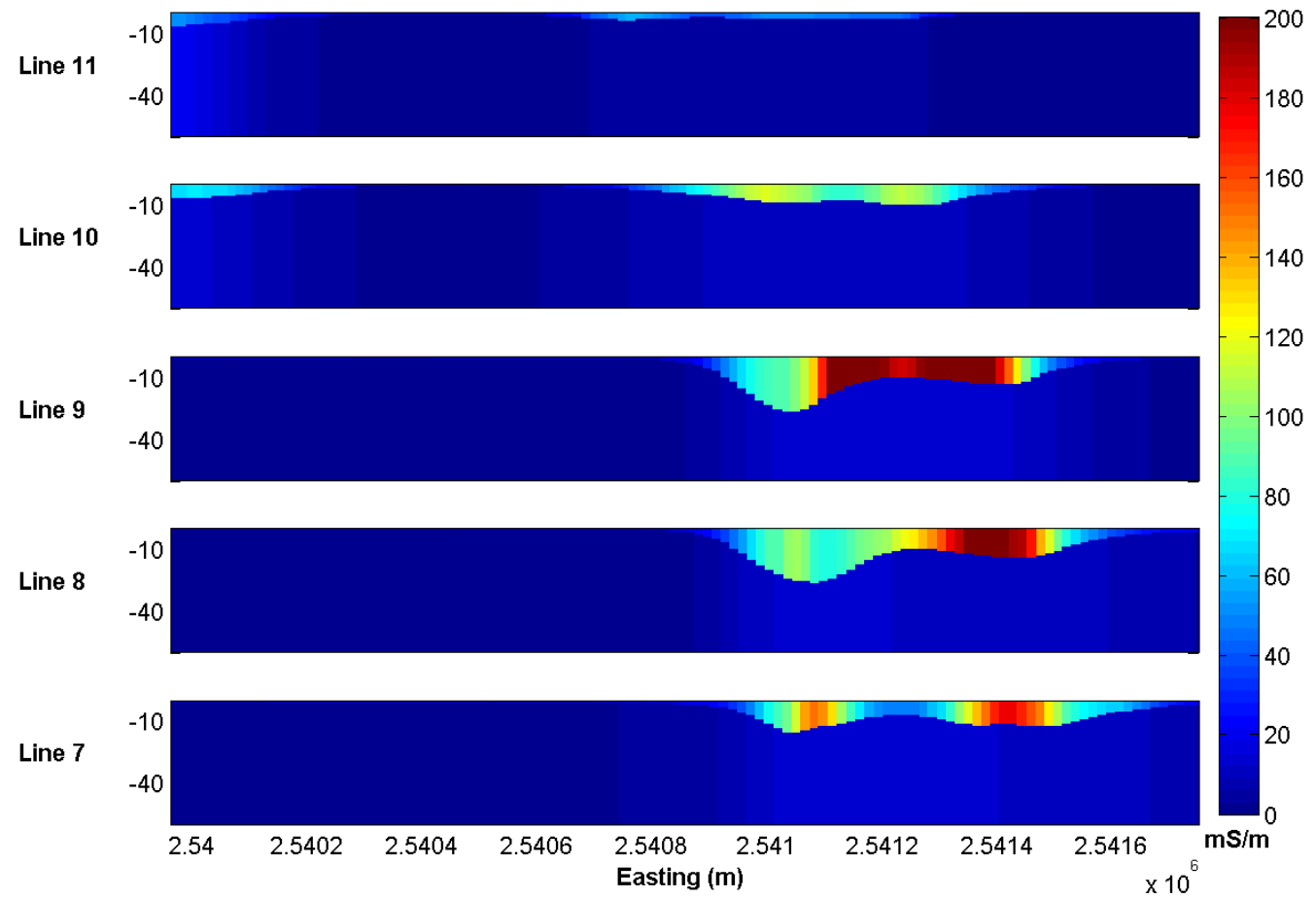

Figure 2. Conductivity cross-sections obtained with LC1DINV for 5 successive lines, $200 \mathrm{~m}$ apart. 


\section{Conclusions}

Inversion of 4-frequency AEM data from the Suurpelto test area in Finland using the LC1DINV algorithm successfully recovers the depth and lateral extent of the near-surface conductive clays. Comparison of this result with those obtained using two different inversion methods shows that LC1DINV is a stable inversion algorithm that produces well-defined layer boundaries and laterally smooth conductivity cross-sections.

\section{Acknowledgements}

This paper is published with the permission of the Executive Director of the British Geological Survey (NERC).

\section{References}

Auken, E., Christiansen, A.V., Jacobsen, B.H., Foged, N., and Sorensen, K.I. [2002] Piecewise 1D laterally constrained inversion of resistivity data. Geophysical Prospecting 53 (4), 497-506.

Beard, L.P. [2000] Comparison of methods for estimating earth resistivity from airborne electromagnetic measurements. Journal of Applied Geophysics 45, 239-259.

Constable, S.C., Parker, R.L., and Constable, C.G. [1987] Occam's inversion: a practical algorithm for generating smooth models from electromagnetic sounding data. Geophysics 67, 289-300.

Fitterman, D.V., and Deszcz-Pan, M. [1998] Helicopter EM mapping of saltwater intrusion in Everglades National Park. Exploration Geophysics 29, 240-243.

Hestenes, M.R., and Stiefel, E. [1952] Methods of conjugate gradients for solving linear systems. J. Res. Natl. Bur. Stand. 49, 409-436.

Huang, H., and Fraser, D.C. [1996] The differential parameter method for multi-frequency airborne resistivity mapping. Geophysics 55, 1327-1337.

Kurimo, M., Leinonen, V., Suppala, I., Lahti, M., and Cuss, R.J. [2006] JAC airborne EM hardware: current activities. Hannover AEM Workshop, Expanded Abstract, 19-23.

Paterson, N.R., and Redford, S.W. [1986] Inversion of airborne electromagnetic data for overburden mapping and groundwater exploration. Airborne resistivity mapping, G.J. Palacky (ed.), 39-48. Geological Survey of Canada.

Poikonen, A., Sulkanen, K., Oksama, M., and Suppala, I. [1998] Novel dual frequency fixed wing airborne EM system of Geological Survey of Finland (GTK). Exploration Geophysics 29, 46-51.

Sengpiel, K-P., and Siemon, B. [1998] Examples of 1D inversion of multifrequency HEM data from 3-D resistivity distribution. Exploration Geophysics 29, 133-141.

Smith, T., Hoversten, M., Gasperikova, E., and Morrison, F. [1999] Sharp boundary inversion of 2D magnetotelluric data. Geophysical Prospecting 47 (4), 469-486. 